\title{
Self-Transducing Silicon Nanowire Electromechanical Systems at Room Temperature
}

2008

Vol. 8, No. 6 1756-1761

\author{
Rongrui He, ${ }^{\dagger, \ddagger}$ X. L. Feng, ${ }^{\ddagger, \S}$ M. L. Roukes, ${ }^{\star, \S}$ and Peidong Yang ${ }^{\star, \dagger, \|}$ \\ Department of Chemistry, University of California, Berkeley, California 94720, \\ Materials Science Division, Lawrence Berkeley National Laboratory, Berkeley, \\ California 94720, and Kavli Nanoscience Institute, California Institute of Technology, \\ Mail Code 114-36, Pasadena, California 91125
}

Received April 15, 2008

\begin{abstract}
Electronic readout of the motions of genuinely nanoscale mechanical devices at room temperature imposes an important challenge for the integration and application of nanoelectromechanical systems (NEMS). Here, we report the first experiments on piezoresistively transduced very high frequency Si nanowire (SiNW) resonators with on-chip electronic actuation at room temperature. We have demonstrated that, for very thin $(\sim 90 \mathrm{~nm}$ down to $\sim 30 \mathrm{~nm})$ SiNWs, their time-varying strain can be exploited for self-transducing the devices' resonant motions at frequencies as high as $\sim 100 \mathrm{MHz}$. The strain of wire elongation, which is only second-order in doubly clamped structures, enables efficient displacement transducer because of the enhanced piezoresistance effect in these SiNWs. This intrinsically integrated transducer is uniquely suited for a class of very thin wires and beams where metallization and multilayer complex patterning on devices become impractical. The $\mathbf{3 0}$ $\mathrm{nm}$ thin SiNW NEMS offer exceptional mass sensitivities in the subzeptogram range. This demonstration makes it promising to advance toward NEMS sensors based on ultrathin and even molecular-scale SiNWs, and their monolithic integration with microelectronics on the same chip.
\end{abstract}

Recent advances in resonant nanoelectromechanical systems (NEMS) promise a variety of important applications, including ultrasensitive mass ${ }^{1-5}$ and force ${ }^{6}$ sensing, ultralow-power radio frequency (RF) signal generation and timing, ${ }^{7,8}$ and fundamental studies in quantum measurement. ${ }^{9,10}$ In the pursuit of very small and yet robust and high-performance devices, bottom-up chemically synthesized nanowires have emerged as attractive material candidates for NEMS resonators because of their ultimately miniaturized sizes and atomically smooth surfaces. ${ }^{11}$ The exploitation of bottomup nanowire-based NEMS has been hindered by the difficulty of integrating them into functional devices. This is mainly because most existing chemical synthesis techniques do not spontaneously provide nanowires with controllable mechanical degrees of freedom and thus require nontrivial postgrowth assembly of nanowires, which often limits device yield. We have recently developed a technique to directly grow doubly clamped, single-crystalline $<111>$ SiNWs in lithographically defined microtrenches on silicon-on-insulator

\footnotetext{
* Corresponding author. E-mail: roukes@caltech.edu (M.L.R.) and p_yang@berkeley.edu (P.Y.).

${ }^{\dagger}$ University of California.

* These authors contributed equally to this work.

$\S$ California Institute of Technology.

"Lawrence Berkeley National Laboratory.
}

(SOI) substrates ${ }^{12}$ (Figure 1a). Such naturally suspended nanowires immediately function as high frequency NEMS resonators without the need of further device processing. ${ }^{13}$ On the basis of this hybrid growth process, we have now engineered to produce typical SiNWs with sub-100 nm widths, down to $30 \mathrm{~nm}$. Combined with typical trench width (hence SiNW length) tailored from $\sim 5 \mu \mathrm{m}$ to $\sim 0.5 \mu \mathrm{m}$, we have designed devices with fundamental resonances scaled up to very high frequency (VHF, 30-300 MHz), ultra-high frequency (UHF, $300 \mathrm{MHz}-1 \mathrm{GHz}$ ), and microwave (>1 $\mathrm{GHz}$ ) ranges. Controlling of the process, such as the areas of the $\{111\}$ trench walls and the number density of catalyst particles, ${ }^{12}$ enables us to attain arrays of self-aligned SiNW resonators.

For these nanowires to realize their promising application potentials, on-chip, integrated transduction schemes are highly desirable. To date, significant effort has been made to develop displacement transducers,${ }^{14}$ as electronic readout of miniscule motions for NEMS has been a central challenge in this field. Some techniques have been demonstrated with remarkable efficiency and sensitivity. For example, the magnetomotive transduction readily reads out microwave NEMS resonances $;{ }^{15}$ and the single electron transistor (SET) is capable of sensing displacement near the quantum limit. ${ }^{9}$ 


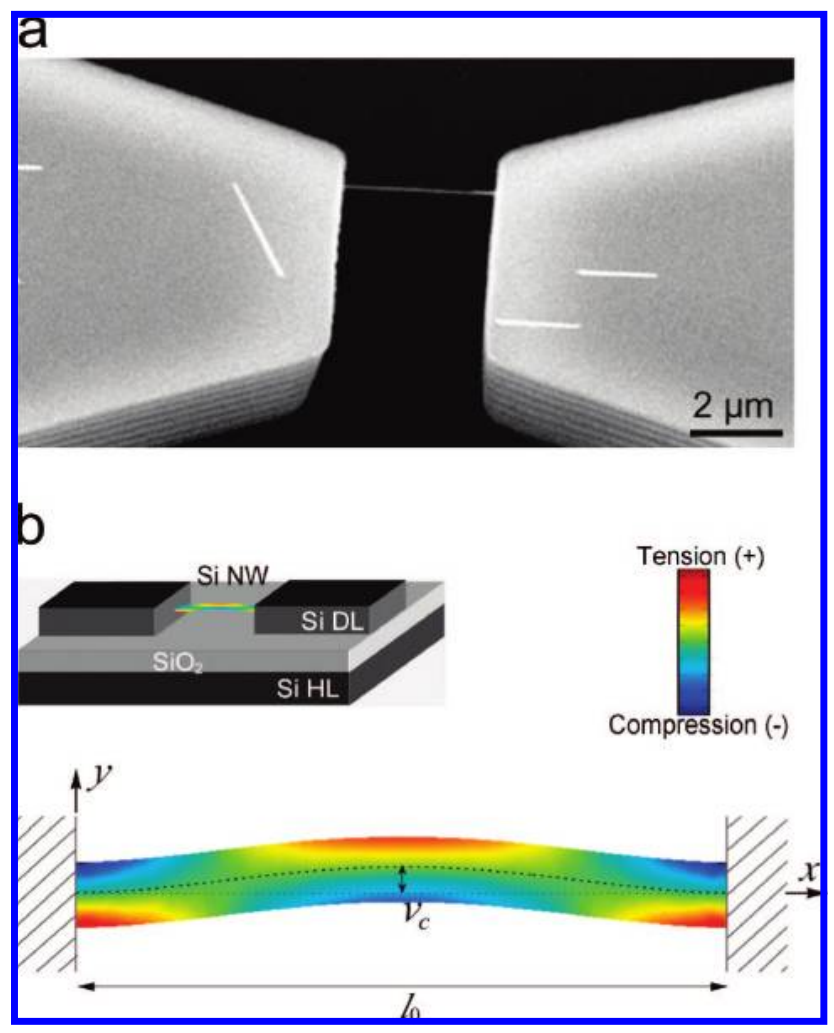

Figure 1. Device architecture and self-transducing principle of $\mathrm{Si}$ nanowire resonators. (a) Scanning electron microscopy (SEM) image of a $\mathrm{Si}$ nanowire resonator grown by chemical vapor deposition. The doubly clamped device is suspended in a trench fabricated on a silicon-on-insulator (SOI) substrate. (b) The top left diagram shows the nanowire-SOI device architecture; DL stands for device layer and HL stands for handle layer. Nanowire deflection is illustrated with a strain color map (encoded as shown on the top right). The bottom diagram illustrates the resonance mode shape and the distribution of the longitudinal strain (along the $x$ direction) for the vibrating doubly clamped nanowire (also color mapped), simulated by finite element analysis; the transverse displacement is exaggerated.

Such schemes, however, are usually associated with stringent system operating conditions such as high magnetic fields and low temperatures. At room temperature, optical interferometry detection ${ }^{16,17}$ is convenient and ubiquitous; but its sensitivity for very small devices is limited by diffraction. ${ }^{17,18}$ Alternatively, near-field optical techniques are also being explored, as recently demonstrated in the readout of NEMS motions utilizing the evanescent-wave coupling between suspended waveguides. ${ }^{19}$ Capacitive detection, a common scheme widely used for MEMS resonators, ${ }^{8}$ has the advantages of being inherently on-chip and all-electronic. It also faces challenges in the size regime of NEMS because the sensing capacitances rapidly decrease (down to sub-aF levels) and result in huge motional impedances (up to $\mathrm{M} \Omega$ and $\mathrm{G} \Omega$ levels) at RF. This imposes significant impedance mismatch (with $50 \Omega$ ) and prohibits efficient RF readout. An important progress has recently been made by using inductor-capacitor impedance matching networks to evade the above issues in capacitive detection of NEMS. ${ }^{20}$ This useful technique and similar concepts have also been employed earlier in the development of ultrasensitive RF-SET ${ }^{21}$ and, more recently, the RF scanning tunneling microscope (STM). ${ }^{22}$ Despite these achievements, room-temperature, on-chip sensitive readout of VHF and UHF NEMS made of sub-100 nm thin beams and wires still remains challenging. In fact, recent demonstrations of optical and capacitive detections have primarily been for $<30 \mathrm{MHz}$ beams with $>200 \mathrm{~nm}$ widths. ${ }^{17-20}$

For very thin SiNW-based NEMS, we seek to develop novel integrated transduction schemes that exploit the unique intrinsic properties of such structures. These must be complementary with realizable device configurations and established growth processes. ${ }^{12}$ Ingeniously engineering the strains in these fine SiNWs may be key to creating new displacement transducers, similar to the important roles of detailed strain engineering in complex MEMS structures. ${ }^{23}$ By introducing static longitudinal strains, we have discovered that the suspended SiNWs exhibit significantly enhanced piezoresistance effect compared with bulk $\mathrm{Si}^{24}$ As an example, for the SiNWs with the highest gauge factor $\left(G_{P R}\right)$ observed, that is, $G_{P R} \sim 5000\left(G_{P R}\right.$ is defined as the relative change in resistance per unit longitudinal strain $\epsilon_{l}$ ), a $0.01 \%$ axial elongation can induce a $50 \%$ change in its resistance $\left(\Delta R / R_{0}=G_{P R^{*}} \epsilon_{l} \sim 50 \%\right)$. We demonstrate here that the nanowire resistance change based on this effect provides excellent strain transducers that are naturally integrated within the nanowires themselves.

The most significant strain in a vibrating doubly clamped beam or wire structure is the longitudinal strain $\epsilon_{x}^{b}$ generated by the bending moment. However, with tensile and compressive strains of the same magnitude distributed on the opposite sides of the neutral surface (Figure 1b), there will be a zero change in total resistance. To utilize this strain, usually one has to fabricate a piezoresistor loop on the beam surface to collect strain of the same sign. ${ }^{25}$ This approach can lead to additional energy dissipation and is subject to formidable fabrication difficulties when patterning piezoresistors on sub$100 \mathrm{~nm}$ thin beams and wires. On the other hand, the lengthening of a deflected wire produces a nonvanishing longitudinal strain $\epsilon_{l}$ through the wire, which can be calculated from the transverse displacement $v(x)$ as

$$
\epsilon_{l}=\frac{\Delta l}{l_{0}} \approx \frac{1}{2 l_{0}} \int_{0}^{l_{0}}\left(\frac{\mathrm{d} v}{\mathrm{~d} x}\right)^{2} \mathrm{~d} x=2.44\left(\frac{v_{c}}{l_{0}}\right)^{2}
$$

where $l_{0}$ is the original length of the wire and $v_{\mathrm{c}}$ is the displacement at the wire center $x=l_{0} / 2$ (Figure 1b). ${ }^{26}$ This strain is of small magnitude for slightly deflected wires, because of its quadratic dependence on the displacement. In the case of SiNWs, however, the large gauge factors compensate this and readily provide a detectable change in resistance. The SiNWs studied here have resistivity of $\sim 0.01$ $\Omega \cdot \mathrm{cm}$ and widths ranging from 30 to $90 \mathrm{~nm}$; their gauge factors are around 2000 (ref 24). As an estimate, with $v_{\mathrm{c}} \sim$ $1 \mathrm{~nm}$ and $l_{0} \sim 1 \mu \mathrm{m}, \Delta R / R_{0}=2.44 G_{\mathrm{PR}}\left(v_{\mathrm{c}} / l_{0}\right)^{2}$ is on the order of $5 \times 10^{-3}$, which can be easily monitored electronically. For a nanowire resonator being driven into harmonic vibration, $v_{\mathrm{c}}$ is a function of frequency $\omega$ and time $t: v_{c}(\omega, t)$ $=a(\omega) \cos (\omega t+\varphi)$, where $\mathrm{a}(\omega)$ is the amplitude and $\varphi$ is the phase. As a direct consequence of eq 1, the resistance change will be proportional to the square of displacement and vary in the doubled frequency $2 \omega$ (i.e., the wire is lengthened twice per cycle of vibration): 


$$
\frac{\Delta R(\omega, t)}{R_{0}} \propto G_{P R} a^{2}(\omega) \cos (2 \omega t+2 \varphi)
$$

This change in resistance can be measured by monitoring the voltage change in a frequency down-conversion circuit with the nanowire device serving as a nanomechanical mixer (Figure 2a and Figure 3a). The SiNW is excited into motion at $\omega$, while the ac bias voltage is set as $V_{b} \cos (2 \omega t+\Delta \omega t)$ to accommodate the $2 \omega$ varying $\Delta R$. The piezoresistive signal is time-varying at the intermediate frequency $\Delta \omega$ and thus can be readily measured via lock-in detection. The amplitude of the measured voltage signal $V_{s}$ is expected to vary with sweeping excitation frequency $\omega$ :

$$
V_{s}(\omega) \propto \frac{\Delta R}{R_{0}} V_{b} \propto \frac{1}{\left(\omega_{0}^{2}-\omega^{2}\right)^{2}+\omega_{0}^{4} / Q^{2}} G_{P R} F^{2} V_{b}
$$

where the frequency-domain response of the device motion amplitude is described by a damped harmonic resonator model, ${ }^{27}$ with $\omega_{0}$ as the fundamental resonance frequency, $Q$ as the quality factor, and $F$ as the force per unit length applied on the resonator. We call this scheme the two-omega $(2 \omega)$ piezoresistive detection, in which the key feature is that the resistance and voltage signals are proportional to the square of device displacement. We note that this is different from the piezoresistive detection based on first-order strain (due to bending), ${ }^{25,28}$ where the bias is close to the vibrating frequency (one-omega detection).

To demonstrate the principle of this two-omega piezoresistive detection with the intrinsically embedded nanowire strain transducers, we first excite the nanowire into resonances via direct mechanical coupling. The device chip is mounted on a piezoelectric ceramic disk with a thicknessmode resonance frequency of $\sim 18 \mathrm{MHz}$. An ac drive voltage, $V_{d}^{A C} \cos (\omega t)$, is applied to vibrate the disk, which in turn actuates the nanowires on the chip (Figure 2a). Nanowire resonators with expected resonance frequencies in the range of $\sim 20-50 \mathrm{MHz}$ are chosen to take advantage of the efficient piezoelectric disk actuation in that range. The measurements are carried out at room temperature, in a vacuum chamber with pressures of $\sim 10^{-3}$ Torr.

Figure $2 \mathrm{~b}$ shows one of the resonance curves measured from an $80 \mathrm{~nm}$ thick, $4.6 \mu \mathrm{m}$ long Si nanowire, with a twoterminal dc resistance of $106 \mathrm{k} \Omega$. The resonance peak is readily detected at $29.9 \mathrm{MHz}$ with a measured quality factor $Q$ of $\sim 800$. Here, we note that throughout this work the signal amplitudes are all referred to the input of the first stage electronic amplification, at the point right after the SiNW transducers. The resonance curve fits well to the Lorentzian function depicted in eq 3 , while apparent deviation occurs in the attempt of fitting the data to the square root of the Lorentzian (i.e., $\sim 1 / \sqrt{\left(\omega_{0}^{2}-\omega^{2}\right)^{2}+\omega_{0}^{4} / Q^{2}}$ ). Since the resonant mechanical vibration displacement follows the square root of the Lorentzian function, this clearly validates the fact that the detected signals are proportional to the square of the displacement in our measurements (eq 2). This is in contrast to many other transduction schemes in which the voltage signal varies linearly with the displacement. ${ }^{13-20,25,28}$ The device can be driven into nonlinear response with application of sufficient drive, as shown for a $22 \mathrm{MHz}$ device of $88 \mathrm{~nm}$ thick, $4.9 \mu \mathrm{m}$ long, $11 \mathrm{k} \Omega$ in resistance, with a

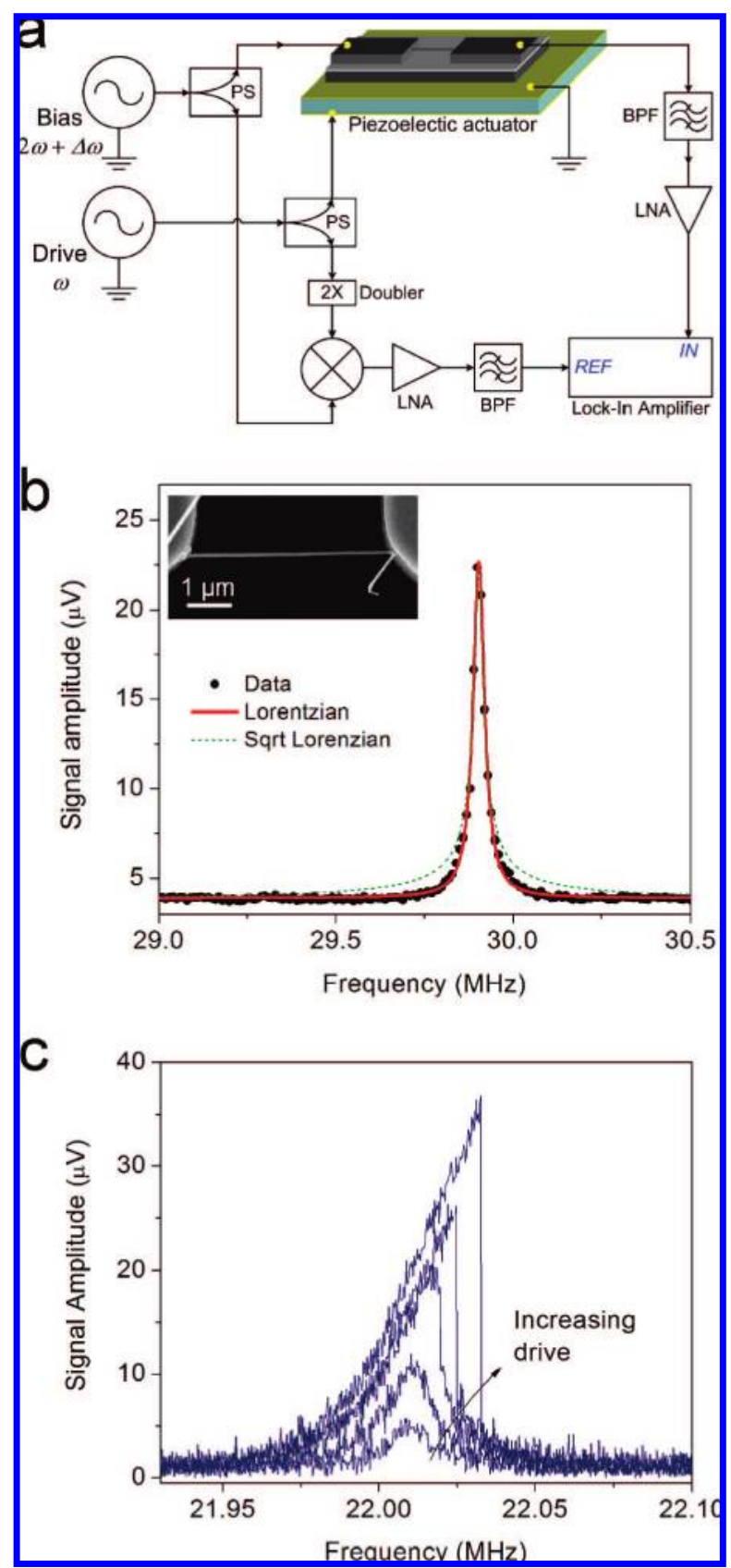

Figure 2. High frequency Si nanowire resonators with piezoelectric actuation and piezoresistive self-detection. (a) Schematic diagram of the measurement circuit. PS denotes power splitter; $2 X$ denotes frequency doubler; BPF denotes band-pass filter; and LNA denotes low noise amplifier. (b) An $80 \mathrm{~nm}$ thick, $4.6 \mu \mathrm{m}$ long nanowire resonator, with its morphology shown in the inset. The data (solid circle) are taken at drive voltage $V_{d}^{A C}=0.58 \mathrm{~V}$ and bias voltage $V_{b}$ $=0.16 \mathrm{~V}$. The red curve is the best Lorentzian fit to the data of the amplitude signal, and the dashed green curve is a square root Lorentzian fit to the same data. Both are the best achievable fits given the restraints of the peak and background level of the resonance signal. (c) Nonlinear vibration from an $88 \mathrm{~nm}$ thick, 4.9 $\mu \mathrm{m}$ long nanowire. The curves are corresponding to $V_{d}^{A C}=0.32$, $0.50,0.63,0.71$, and $0.79 \mathrm{~V}$ respectively, with the same bias of $V_{b}$ $=0.25 \mathrm{~V}$

measured $Q \sim 1200$ (Figure 2c). This onset of nonlinearity, characterized by the frequency stiffening and bistability, arises from the effect of increasing tension built up in the wire at large vibration amplitudes. ${ }^{29}$ Given our method of 


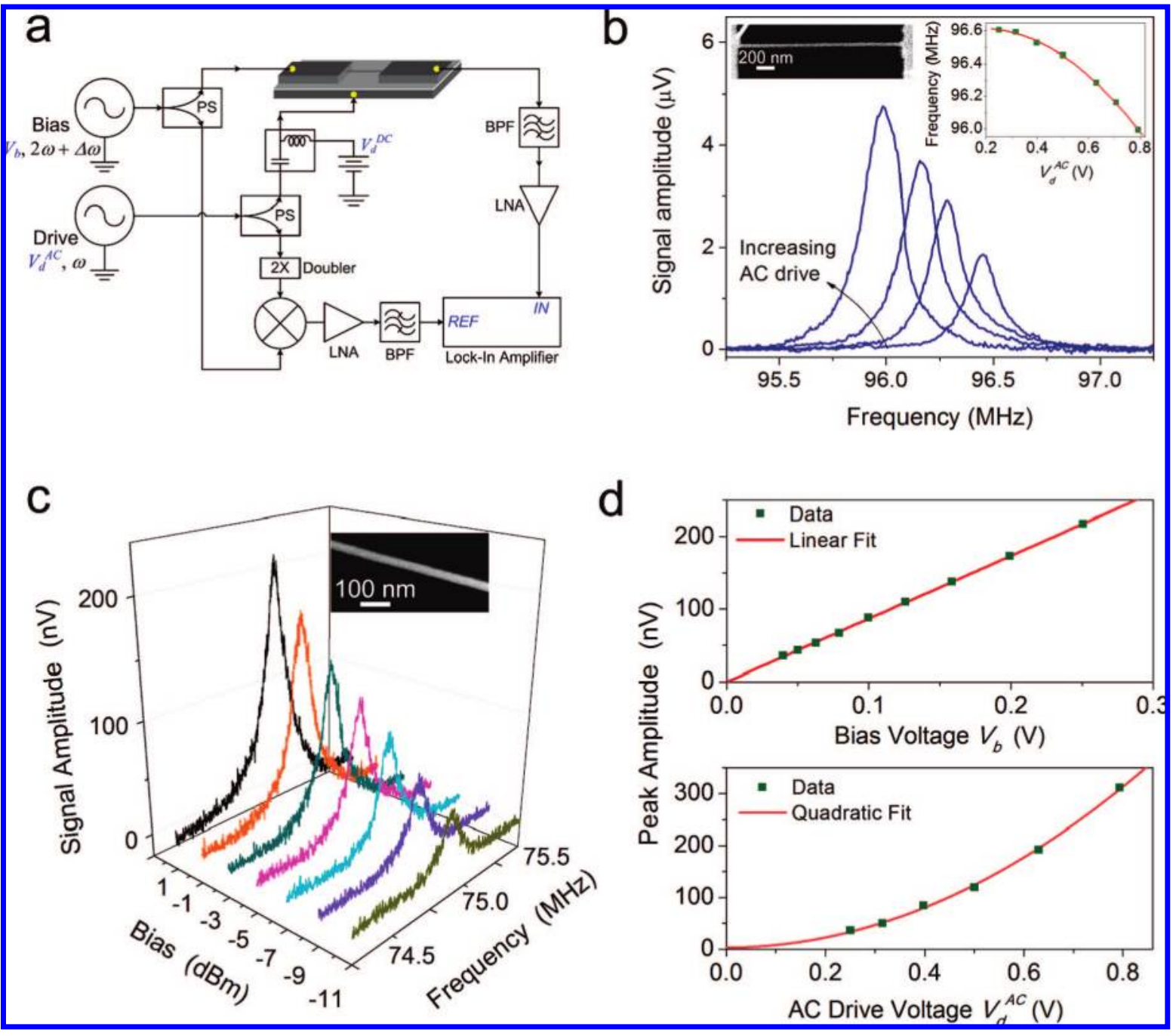

Figure 3. Very high frequency Si nanowire resonators with fully integrated electrostatic actuation and piezoresistive self-detection. (a) Schematic diagram of bias and drive circuitry. (b) Device performance of a $40 \mathrm{~nm}$ thick, $96 \mathrm{MHz}$ nanowire resonator with a quality factor $Q$ of $\sim 550$. It is $1.8 \mu \mathrm{m}$ long and has a dc resistance of $80 \mathrm{k} \Omega$. The ac drive is set at $V_{d}^{A C}=0.50,0.63,0.71$, and $0.79 \mathrm{~V}$ for the curves, respectively, with the dc voltage fixed at $V_{d} D C=0.2 \mathrm{~V}$. The left inset shows the SEM image of the device, and the right inset shows the drive dependence of the resonance frequency. (c) Performance of a $30 \mathrm{~nm}$ thick, $75 \mathrm{MHz}$ Si nanowire resonator with $Q \sim 700$. Its length is $1.8 \mu \mathrm{m}$, and its dc resistance is $300 \mathrm{k} \Omega$. The curves are taken at different bias voltages with the same drive. The inset shows the SEM image. (d) The bias and drive voltage dependence of peak amplitude for the $30 \mathrm{~nm}$ device.

mechanical actuation, no other mechanisms, except for the piezoresistance effect, can be responsible for the nanowire's resistance change at twice of its vibrating frequency. This further verifies that the nanowire's intrinsic piezoresistance effect serves as an efficient mechanism for integrated transduction.

The efficiency and sensitivity of this intrinsic transduction scheme is noteworthy. The efficiency of a detection scheme is, in general and intuitively, how big the signal from the device of interest is, compared with the response (background) induced by implementing the scheme itself. We characterize the detection efficiency by the signal-tobackground ratio (SBR) in $\mathrm{dB} .{ }^{13}$ In the present work, the background (i.e., off-resonance) response mainly originates from parasitic effects and cross talk, for example, direct feedthrough of RF excitation from the drive and bias ports to the detection port and their frequency down-conversion products. Typically, very large SBR's, up to $15-20 \mathrm{~dB}$ on resonance, have been achieved with excitation levels near the onset of nonlinearity ${ }^{29}$ (Figure 2b,c). The sensitivity of a detection scheme, how small a signal the scheme is able to resolve, is limited by the detection noise floor. The achievable noise floor is currently limited by the device resistance's thermal (Johnson) noise, which varies from device to device with their resistances. We have achieved signal-to-noise ratios (SNR's) in the range from $\sim 250$ to $\sim 630$, corresponding to an operating dynamic range ${ }^{29}$ from $\sim 48 \mathrm{~dB}$ to $\sim 56 \mathrm{~dB}$.

For practical applications, on-chip integrated actuation of mechanical response is desirable. We find that electrostatic actuation is an efficient mechanism for exciting our nanowire NEMS. Here, the SOI structures are readily utilized for capacitive coupling to the nanowires with no additional device modifications. The device and oxide layers are made relatively thin $(\sim 400 \mathrm{~nm})$ on the SOI chips so as to obtain sufficiently large coupling capacitance. The driving voltage, 
including both an ac excitation component $V_{d}^{A C} \cos (\omega t)$ and a dc polarization component $V_{d}^{D C}$, is directly applied to the heavily doped handle layer (Figure 3a). This yields a driving force at frequency $\omega$ with its amplitude proportional to $V_{d}^{A C} V_{d}^{D C}$ (ref 30). For detection, the SiNW is again ac biased at $2 \omega+\Delta \omega$ to provide readout of the piezoresistive signal that is quadratically dependent upon the nanowire displacement. This fully integrated excitation-detection scheme is depicted in Figure 3a.

This scheme provides sensitive and efficient detection. Furthermore, it enables detection of the vibrations of nanowires with very small dimensions and VHF operations. For example, Figure $3 \mathrm{~b}$ demonstrates the resonant response at about $96 \mathrm{MHz}$ from a $40 \mathrm{~nm}$ thin nanowire, and Figure $3 \mathrm{c}$ shows data from a $30 \mathrm{~nm}$ thin nanowire resonating at 75 MHz. The resonance characteristics of both devices are investigated under a series of drive and bias voltages. We observe that the resonance frequency decreases with increasing ac drive voltage and follows a quadratic dependence on $V_{d}^{A C}$ (inset of Figure $3 \mathrm{~b}$ ). This frequency down-tuning with increasing $V_{d}^{A C}$ arises because the $V_{d}^{A C}$ driving term contributes to an ac force term that is proportional to $V_{d}^{D C 2}+V_{d}^{A C 2} / 2$, thus reducing the wire's effective stiffness. This acts to shift the frequency downward ${ }^{31}\left(V_{d}^{D C}\right.$ is kept constant and low enough as compared with $V_{d}^{A C}$ in the data shown in Figure 3b). For the $29.9 \mathrm{MHz}$ device shown in Figure 2b, we are able to drive the resonance into nonlinear response using the same electrostatic scheme after removing the underneath piezoelectric actuator. We have observed nonlinear frequency pulling downward (i.e., softening), as well as hysteresis (data not shown). This frequency softening nonlinearity arises from the combination of both quadratic and cubic nonlinearities in the response; ${ }^{32}$ these originate primarily from complex nonlinear electrostatic coupling effects at large amplitudes. This is in contrast to the tension-induced stiffening nonlinearity observed in the piezoelectric actuation experiments (Figure 2c), where we have intentionally eliminated direct electrostatic interactions between the drive and the nanowire. We note that frequency tuning and readily accessible nonlinear response can be useful in controlling frequency instability and deterministic switching between bistable states.

The drive and bias dependence of signal amplitudes are summarized in Figure 3c,d for the $75 \mathrm{MHz}$ device. Peak amplitudes are extracted from Lorentzian fitting after background subtraction. The amplitude shows a linear dependence upon bias voltage and a quadratic dependence upon drive voltage. Note that the fitting curves (in red) both extrapolate to the origin. These relations are consistent with the expectations from eq 3; for example, since the driving force amplitude $F$ at $\omega$ is proportional to $V_{d}^{A C} V_{d}^{D C}$, the measured voltage signal amplitude $V_{s}$ should scale quadratically with $V_{d}^{A C}$ when $V_{d}^{D C}$ is fixed. These results confirm the second order nature of the piezoresistance signal in the $2 \omega$ detection.

We note that the field effect from electrostatic actuation could also modulate (i.e., "gate") the resistance of the semiconductor nanowire. In the present work, this contribu- tion is negligible; the transconductance of these heavily doped nanowires $\left(10^{-9}\right.$ to $10^{-8} \mathrm{~S} / \mathrm{V}$ by measurements) is extremely small and with the relatively large gate distance $(\sim 800 \mathrm{~nm})$, the relative resistance change from the field effect is estimated to be only about $10^{-7}$ to $10^{-6}$. This is 3 orders of magnitude smaller than the changes arising from the piezoresistance effect.

We believe our $30 \mathrm{~nm}$ thick SiNW resonators are the thinnest nanowire NEMS realized to date with a fully integrated self-transducing architecture. We anticipate that sub-10 nm thin SiNW resonators are attainable using Pt catalyst nanoparticles in the growth process. ${ }^{33}$ Generally, a detection scheme will become less efficient for smaller devices as parasitic impedance increases, and the large device resistance would deteriorate the signal transmission. In this case, however, we expect the piezoresistance detection will maintain sensitive even for smaller devices since the gauge factor increase for thinner SiNWs compensates. ${ }^{24}$ The detection scheme demonstrated here is general and will work for other materials with appreciable gauge factors. Our measurements indicate that a gauge factor of $\sim 50$, which is accessible for a range of semiconductor materials, ${ }^{34-37}$ can provide a decent SNR of the order $\sim 10-100$ for a resonance signal approaching the critical amplitude.

The surface quality of the SiNWs is generally wellpreserved in our pristine nanowire-in-microtrench structures. The resonator $Q$ 's are free from the dissipation caused by (i) metallization layers used for other modes of transduction, ${ }^{13}$ (ii) surface roughness, ${ }^{38}$ and (iii) other surface related losses. We believe that the $Q$ 's of 550 to 1200 obtained for these resonators could be further improved with optimized nanowire synthesis. Experiments also show that the quality factors do not drop until pressure reaches $\sim 1$ Torr and are still appreciable (e.g., $Q$ 's $\sim 300$ for $30 \mathrm{~nm}$ thick resonators) around 100 Torr.

The very thin SiNWs with VHF operations demonstrated here are promising for sensors with exceptional sensitivities. For example, the $30 \mathrm{~nm}$ thin, $75 \mathrm{MHz}$ SiNW resonator has a very small effective device mode mass itself, only $M_{\text {eff }} \approx$ $2.5 \mathrm{fg}$. This makes it highly responsive for mass loading, with a large mass responsivity ${ }^{2}$ of $\mathscr{R} \approx 15 \mathrm{~Hz} / \mathrm{zg}(1 \mathrm{zg}=$ $10^{-21} \mathrm{~g}$ ). Combined with a practically achievable fractional frequency fluctuation of $\delta f_{0} / f_{0} \sim 1 \times 10^{-7}(0.1 \mathrm{ppm})$ demonstrated for such VHF NEMS, ${ }^{2,13}$ this device offers a mass resolution of $\delta M \approx 0.5 \mathrm{zg}=500 \mathrm{yg} \approx 300$ Dalton (1 $\mathrm{yg}=10^{-24} \mathrm{~g} \approx 0.6$ Dalton). Such a mass sensitivity is excellent for real-time single-biomolecule mass detection. Sub-10 nm SiNWs ${ }^{11,33}$ should make even more responsive, UHF devices to approach single-Dalton sensitivity. ${ }^{39}$ Moreover, because of the quadratic dependency of the measured signal on the device displacement, the new $2 \omega$ readout scheme developed in this work might also be interesting for quantum nondemolition measurements where it is preferred to directly detect the square of displacement (energy) than the displacement itself. ${ }^{40}$

In summary, we have demonstrated SiNW-based resonant NEMS with on-chip integrated electronic actuation and detection at room temperature. Utilizing these SiNWs for 
frequency conversion and exploiting their intrinsic strong piezoresistive effect, we have developed the new $2 \omega$ piezoresistive detection technique. It readily provides efficient and sensitive readout of VHF devices with progressively shrinking dimensions (from $\sim 100 \mathrm{~nm}$ down to $30 \mathrm{~nm}$ in width) and high impedances in the range from $\sim 1 \mathrm{k} \Omega$ to $\sim 1 \mathrm{M} \Omega$, without need for extra patterning or metallization. Our simple and practical demonstration of integrated VHF SiNW NEMS should engender new applications of integrated nanowire resonators and arrays.

Acknowledgment. This work was supported by the National Science Foundation under Grant EECS 0425914 (NSF-NSEC), MARCO, and by DARPA/SPAWAR under Grant N66001-02-1-8914. X.L.F. thanks S. Stryker for help in the engineering of the experimental apparatus. R.H. and X.L.F. thank R. T. Howe, R. Maboudian, J. S. Aldridge, I. Bargatin, M. D. LaHaye, and M. Li for helpful discussions. We thank UC Berkeley Microlab and Stanford Nanofabrication Facility for the use of their facilities.

\section{References}

(1) Burg, T. P.; Godin, M.; Knudsen, S. M.; Shen, W.; Carlson, G.; Foster, J. S.; Babcock, K.; Manalis, S. R. Nature 2007, 446, 1066.

(2) Yang, Y. T.; Callegari, C.; Feng, X. L.; Ekinci, K. L.; Roukes, M. L. Nano Lett. 2006, 6, 583.

(3) Ilic, B.; Yang, Y.; Craighead, H. G. Appl. Phvs. Lett. 2004, 85, 2604.

(4) Lavrik, N. V.; Sepaniak, M. J.; Datskos, P. G. Rev. Sci. Instrum. 2004, 75, 2229.

(5) Gupta, A.; Akin, D.; Bashir, R. Appl. Phys. Lett. 2004, 84, 1976.

(6) Rugar, D.; Budakian, R.; Mamin, H. J.; Chui, B. W. Nature 2004, $430,329$.

(7) Feng, X. L.; White, C. J.; Hajimiri, A.; Roukes, M. L. Nature Nanotech. 2008, 3, 342.

(8) Nguyen, C. T. C. IEEE Trans. Ultrason. Ferr. Freq. Contr. 2007, 54, 251.

(9) LaHaye, M. D.; Buu, O.; Camarota, B.; Schwab, K. C. Science 2004, 304, 74.

(10) Naik, A.; Buu, O.; LaHaye, M. D.; Armour, A. D.; Clerk, A. A.; Blencowe, M. P.; Schwab, K. C. Nature 2006, 443, 193.

(11) Wu, Y.; Cui, Y.; Huynh, L.; Barrelet, C. J.; Bell, D. C.; Lieber, C. M. Nano Lett. 2004, 4, 433.

(12) He, R.; Gao, D.; Fan, R.; Hochbaum, A. I.; Carraro, C.; Maboudian, R.; Yang, P. Adv. Mater. 2005, 17, 2098.

(13) Feng, X. L.; He, R.; Yang, P.; Roukes, M. L. Nano Lett 2007, 7, 1953.
(14) Ekinci, K. L. Small 2005, 1, 786.

(15) Huang, X. M. H.; Zorman, C. A.; Mehregany, M.; Roukes, M. L. Nature 2003, 421, 496.

(16) Carr, D. W.; Sekaric, L.; Craighead, H. G. J. Vac. Sci. Technol. B 1998, 16, 3821.

(17) Kouh, T.; Karabacak, D.; Kim, D. H.; Ekinci, K. L. Appl. Phvs. Lett. 2005, 86, 013106.

(18) Karabacak, D.; Kouh, T.; Ekinci, K. L. J. Appl. Phvs. 2005, 98, 124309.

(19) De Vlaminck, I.; Roels, J.; Taillaert, D.; Van Thourhout, D.; Baets, R.; Lagae, L.; Borghs, G. Appl. Phvs. Lett. 2007, 90, 233116.

(20) Truitt, P. A.; Hertzberg, J. B.; Huang, C. C.; Ekinci, K. L.; Schwab, K. C. Nano Lett. 2007, 7, 120.

(21) Schoelkopf, R. J.; Wahlgren, P.; Kozhevnikov, A. A.; Delsing, P.; Prober, D. E. Science 1998, 280, 1238.

(22) Kemiktarak, U.; Ndukum, T.; Schwab, K. C.; Ekinci, K. L. Nature 2007, 450, 85 .

(23) Lin, L. W.; Pisano, A. P.; Howe, R. T. J. Microelectromech. Svst. 1997, 6, 313.

(24) He, R.; Yang, P. Nature Nanotech. 2006, 1, 42.

(25) Bargatin, I.; Kozinsky, I.; Roukes, M. L. Appl. Phvs. Lett. 2007, 90, 093116.

(26) Weaver, W., Jr.; Timoshenko, S. P.; Young, D. H. Vibration Problems in Engineering; Wiley: New York, 1990; pp 416-427.

(27) Cleland, A. N.; Roukes, M. L. J. Appl. Phvs. 2002, 92, 2758.

(28) Bargatin, I.; Myers, E. B.; Arlett, J.; Gudlewski, B.; Roukes, M. L. Appl. Phys. Lett. 2005, 86, 133109.

(29) Postma, H. W. C.; Kozinsky, I.; Husain, A.; Roukes, M. L. Appl. Phvs. Lett. 2002, 86, 223105.

(30) Sazonova, V.; Yaish, Y.; Üstünel, H.; Roundy, D.; Arias, T. A.; McEuen, P. L. Nature 2004, 431, 284.

(31) Kozinsky, I.; Postma, H. W. C.; Bargatin, I.; Roukes, M. L. Appl. Phys. Lett. 2006, 88, 253101.

(32) Nayfeh, A. H.; Mook, D. T. Nonlinear Oscillations; Wiley: New York, 1979; Chapter 4.

(33) Garnett, E.; Liang, W.; Yang, P. Adv. Mater. 2007, 19, 2946.

(34) Tilak, V.; Vertiatchikh, A.; Jiang, J.; Reeves, N.; Dasgupta, S. Phvs. Stat. Sol. (C) 2006, 3, 2307.

(35) Aslam, M.; Taher, I.; Masood, A.; Potter, T. J. Appl. Phvs. Lett. 1992, 60, 2923.

(36) Bykhovski, A. D.; Kaminski, V. V.; Shur, M. S.; Chen, Q. C.; Khan, M. A. Appl. Phvs. Lett. 1996, 68, 818.

(37) Hjort, K.; Söderkvist, J.; Schweitz, J.-Å. J. Micromech. Microeng. 1994, $4,1$.

(38) Huang, X. M. H.; Feng, X. L.; Zorman, C. A.; Mehregany, M.; Roukes, M. L. New J. Phys 2005, 7, 247.

(39) Ekinci, K. L.; Yang, Y. T.; Roukes, M. L. J. Appl. Phys. 2004, 95, 2682.

(40) Santamore, D. H.; Doherty, A. C.; Cross, M. C. Phys. Rev. B 2004, $70,144301$.

NL801071W 